\title{
Liriodendron genome sheds light on angiosperm phylogeny and species-pair differentiation
}

\author{
Jinhui Chen ${ }^{1,11 *}$, Zhaodong Hao ${ }^{1}{ }^{1,11}$, Xuanmin Guang ${ }^{2,11}$, Chenxi Zhao ${ }^{2,11}$, Pengkai Wang', \\ Liangjiao Xue ${ }^{1,3}$, Qihui Zhu ${ }^{4}$, Linfeng Yang ${ }^{2}$, Yu Sheng ${ }^{1}$, Yanwei Zhou', Haibin Xu ${ }^{5}$, Hongqing Xie ${ }^{2}$,

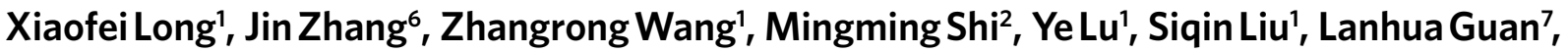 \\ Qianhua Zhu' ${ }^{2}$, Liming Yang ${ }^{5}$, Song Ge ${ }^{8}$, Tielong Cheng ${ }^{5}$, Thomas Laux ${ }^{9}{ }^{9}$, Qiang Gao ${ }^{2}$, Ye Peng ${ }^{5}$, \\ Na Liu ${ }^{2 \star}{ }^{2 \star}$, Sihai Yang ${ }^{10 \star}$ and Jisen Shi ${ }^{1 \star}{ }^{1 \star}$
}

The genus Liriodendron belongs to the family Magnoliaceae, which resides within the magnoliids, an early diverging lineage of the Mesangiospermae. However, the phylogenetic relationship of magnoliids with eudicots and monocots has not been conclusively resolved and thus remains to be determined ${ }^{1-6}$. Liriodendron is a relict lineage from the Tertiary with two distinct species-one East Asian (L. chinense (Hemsley) Sargent) and one eastern North American (L. tulipifera Linn)-identified as a vicariad species pair. However, the genetic divergence and evolutionary trajectories of these species remain to be elucidated at the whole-genome level'. Here, we report the first de novo genome assembly of a plant in the Magnoliaceae, L. chinense. Phylogenetic analyses suggest that magnoliids are sister to the clade consisting of eudicots and monocots, with rapid diversification occurring in the common ancestor of these three lineages. Analyses of population genetic structure indicate that $L$. chinense has diverged into two lineages-the eastern and western groups-in China. While $L$. tulipifera in North America is genetically positioned between the two $L$. chinense groups, it is closer to the eastern group. This result is consistent with phenotypic observations that suggest that the eastern and western groups of China may have diverged long ago, possibly before the intercontinental differentiation between $L$. chinense and $L$. tulipifera. Genetic diversity analyses show that $L$. chinense has tenfold higher genetic diversity than $L$. tulipifera, suggesting that the complicated regions comprising east-west-orientated mountains and the Yangtze river basin (especially near $30^{\circ} \mathrm{N}$ latitude) in East Asia offered more successful refugia than the south-northorientated mountain valleys in eastern North America during the Quaternary glacial period.

The Magnoliaceae, a family in the order Magnoliales, is an early diverging lineage of the Mesangiospermae (core angiosperms) ${ }^{8}$, and thus, it possesses a crucial phylogenetic position for better understanding the evolution of the extant flowering plants. However, the relationships among magnoliids, eudicots, and monocots have not been conclusively resolved despite previous valuable attempts ${ }^{2,5,6}$. The Liriodendron genus, which belongs to the subfamily Liriodendroideae of the Magnoliaceae, consisted of several species distributed throughout the Northern Hemisphere until the Late Tertiary, but now comprises only of a pair of sister species with a classic intercontinental disjunction distribution: one in East Asia (L.chinense) and the other in eastern North America (L. tulipifera). These two Tertiary relict Liriodendron species have been suggested to have diverged during the middle to late Miocene $e^{7,9}$, a reflection of range restrictions resulting from extinctions in the late Cenozoic ${ }^{10}$. Moreover, this pair of species is a perfect verification of the second prediction of the geographic speciation theory, which was proposed to explain the origin of species $^{11,12}$.

Here, we combined three different sequencing technologies (that is, short-read sequencing, long-read sequencing and optical mapping) to de novo assemble the L chinense genome. First, we achieved $\sim 327.11$ gigabases $(\mathrm{Gb})$ of clean Illumina paired-end reads (Supplementary Table 1), $\sim 147.89 \mathrm{~Gb}$ of corrected PacBio long reads (length longer than 2 kilobases $(\mathrm{kb})$; Supplementary Table 2) and $\sim 315.41 \mathrm{~Gb}$ of Bionano genome map data (Supplementary Table 3). We estimated the genome size to be $1.75 \mathrm{~Gb}$ based on Illumina data (Supplementary Fig. 1 and Supplementary Table 4), which was consistent with the estimation of $\sim 1.8 \mathrm{~Gb}$ using flow cytometry (Supplementary Note). Then, we assembled the genome of Liriodendron into 4,624 contigs with an N50 length of 1.43 megabases $(\mathrm{Mb})$ using Falcon (Supplementary Table 5). Furthermore, this assembly of long reads was integrated with a Bionano optical map to create a hybrid assembly consisting of 3,711 scaffolds totalling $1.74 \mathrm{~Gb}$ with an N50 length of $3.53 \mathrm{Mb}$ (Supplementary Table 5). Finally, we anchored 529 scaffolds totalling $\sim 1.37 \mathrm{~Gb}$ to a genetic map with 19 linkage groups, using a total of 1,576 microsatellite markers (Supplementary Fig. 2 and Supplementary Table 6). A high-confidence set of 35,269 gene models was constructed using the genome annotation pipeline MAKER (Supplementary Fig. 3), with $83.59 \%$ of genes being assigned putative functional annotations (Supplementary Table 7). To assess the quality of the

'Key Laboratory of Forest Genetics and Biotechnology, Ministry of Education of China, Co-Innovation Center for the Sustainable Forestry in Southern China, Nanjing Forestry University, Nanjing, China. ${ }^{2}$ BGI Genomics, BGI-Shenzhen, Shenzhen, China. ${ }^{3}$ Warnell School of Forestry and Natural Resources, University of Georgia, Athens, GA, USA. ${ }^{4}$ The Jackson Laboratory for Genomic Medicine, Farmington, CT, USA. ${ }^{5}$ College of Biology and the Environment, Nanjing Forestry University, Nanjing, China. ${ }^{6}$ Department of Surgical and Radiological Sciences, Schools of Veterinary Medicine and Medicine, University of California, Davis, Davis, CA, USA. ' General Station of Forest Seedlings of Hubei Provincial Forestry Department, Wuhan, China. ${ }^{8}$ Institute of Botany, Chinese Academy of Sciences, Beijing, China. 'BIOSS Centre for Biological Signalling Studies, Faculty of Biology, Albert-Ludwigs-Universität Freiburg, Freiburg, Germany. ${ }^{10}$ State Key Laboratory of Pharmaceutical Biotechnology, School of Life Sciences, Nanjing University, Nanjing, China. ${ }^{11 T h e s e}$ authors contributed equally: Jinhui Chen, Zhaodong Hao, Xuanmin Guang, Chenxi Zhao. *e-mail: chenjh@njfu.edu.cn; naliu@bgi.com; sihaiyang@nju.edu.cn; jshi@njfu.edu.cn 
assembly, we compared ten bacterial artificial chromosomes (BACs), in which potential repeat regions were masked (Supplementary Note), with assembled scaffolds, resulting in an average coverage of 99.75\% (Supplementary Fig. 4). Of all 66,934 unigenes (>200 base pairs (bp)) assembled de novo by RNA sequencing (RNA-Seq), more than $90 \%$ had a length coverage of greater than $90 \%$ within a single scaffold (Supplementary Table 8). In addition, 1,300 (90.28\%) genes of the BUSCO plant set were covered by the Liriodendron genome (Supplementary Table 9).

The genome size of $L$. chinense is larger than those of most sequenced angiosperms (Supplementary Fig. 5). We further investigated two pertinent aspects of genome evolution-whole-genome duplication (WGD) events and transposable element bursts-both of which have had profound effects on plant genome evolution ${ }^{13}$. The fraction of synonymous substitutions per synonymous site $\left(K_{\mathrm{s}}\right)$ distributions of paralogues in the Liriodendron genome and transcriptome clearly illustrate the occurrence of a single WGD event experienced by Liriodendron (Fig. 1a,b). It has been firmly established that whole-genome triplication (mechanistically originating as two successive WGDs) occurred in the grape ${ }^{14}$, and there is no evidence for lineage-specific polyploidy events in Amborella ${ }^{15}$. By performing a comparative genomic analysis of Vitis with Amborella and Liriodendron, we identified 3:1 and 3:2 syntenic depth ratios in the Vitis-Amborella (Supplementary Fig. 6) and Vitis-Liriodendron (Fig. 1c and Supplementary Fig. 7) comparisons, respectively. Furthermore, we mapped the complete repertoire of 1-2-3 orthologous regions in the Amborella-Liriodendron-Vitis genome comparison (Fig. 1d,e). Thus, from these data, we conclude that a single Liriodendron lineage-specific WGD event occurred, consistent with the results of the fourfold synonymous third-codon transversion position analysis (Supplementary Fig. 8). We speculated that the Liriodendron WGD event occurred approximately 116 million years ago $(\mathrm{Ma})$ with a synonymous substitution rate of $3.02 \times 10^{-9}$ synonymous substitutions $\mathrm{yr}^{-1}$ (ref. ${ }^{16}$ ). Considering the possibly overestimated synonymous substitution rate $^{16}$ and the divergence time of 113-128 Ma between the families Magnoliaceae and Lauraceae ${ }^{17}$, the WGD detected in the Liriodendron genome probably predated the separation of these two families.

Transposable elements account for $61.64 \%$ of the Liriodendron genome (Supplementary Tables 10 and 11). Long terminal repeat (LTR) retrotransposons are the most abundant transposable element, representing $56.25 \%$ of the assembly (Supplementary Table 11). Among the LTR retrotransposons, Gypsy elements are much more abundant than Copia elements (Supplementary Table 12 and Supplementary Fig. 9). In addition, transposable elements are unevenly distributed across the Liriodendron genome and tend to accumulate in intergenic regions rather than genic regions and regions adjoining genes (Supplementary Fig. 10), probably as a result of natural selection due to the potential detrimental effects of transposable elements on gene expression ${ }^{18}$. With respect to the genic regions, transposable elements have an unequal distribution between exons and introns, and there is an obvious bias towards transposable element accumulation in introns compared with exons (Supplementary Fig. 11), consistent with the natural selection hypothesis, although introns may play an important role in gene expression $^{19}$. Furthermore, long interspersed nuclear element-1 has an abnormally high rate of accumulation in genic regions, in contrast with the pattern shown by other transposable elements (Supplementary Fig. 12 and Supplementary Table 13). Moreover, we analysed the divergence time distribution for all LTRs in the Liriodendron genome and found a $K_{\mathrm{s}}$ peak at 0.05 (Supplementary Fig. 13). We assumed an intergenic nucleotide substitution rate of $1.51 \times 10^{-9}$ that was roughly twice as low as that within the genic regions (Supplementary Note), resulting in an insertion time of $\sim 16 \mathrm{Ma}$. Overall, these results show that an ancient WGD event that occurred approximately $116 \mathrm{Ma}$, followed by a more recent burst of transposable element insertion that occurred approximately $16 \mathrm{Ma}$, have both contributed to the expansion of the Liriodendron genome.

Some features of the Liriodendron phenotype are typical of both monocots and eudicots (Fig. 2a), which is consistent with the obscure phylogenetic relationships among magnoliids, monocots and eudicots. To investigate which of the three previously proposed tree topologies is most likely to be true (that is: (1) ((monocots, (eudicots, magnoliids)), basal angiosperm); (2) ((eudicots, (monocots, magnoliids)), basal angiosperm); or (3) ((magnoliids, (monocots, eudicots)), basal angiosperm) (Supplementary Table 14)), we selected an additional six eudicots, six monocots, three magnoliids and one basal angiosperm, with one gymnosperm being the outgroup (Supplementary Fig. 14), to construct individual orthogroups. In this way, we could use as many gene families as possible to depict a broad picture of the phylogeny. After careful evaluation and selection (Supplementary Note), we finally obtained 502 low-copy orthogroups, with 172 orthogroups (34.26\%) supporting topology I, 155 orthogroups $(30.88 \%)$ supporting topology II and the final 175 orthogroups (34.86\%) supporting topology III (Fig. 2b), with no statistically significant difference among the three topologies $\left(\chi^{2}=1.3904 ; P=0.4990\right)$. Based on these 502 low-copy orthogroups, quantification of differences in gene-wise log-likelihood scores ( $\Delta$ GLS) among these three alternative topologies ${ }^{20}$ showed an equal distribution of phylogenetic signals for each topology at the gene level (Supplementary Fig. 16). Further excluding orthogroups whose $\Delta$ GLS values were outliers (Supplementary Note), we obtained 481 low-copy orthogroups, with a lack of statistical significance among the orthogroups supporting each of the three alternative topologies (Fig. $2 b ; \chi^{2}=0.2162 ; P=0.8975$ ). These results explain why all three possible topologies have been observed in previous studies using different datasets (Supplementary Table 14) and suggest that rapid diversification occurred in the common ancestor of magnoliids, eudicots and monocots, which might be responsible for the phylogenetic incongruence in previous studies.

To further confirm the Liriodendron phylogeny, a coalescentbased species tree was constructed using the 502-orthogroup dataset, and this tree supported topology III with low bootstrap support (Supplementary Fig. 17a). Additionally, we performed coalescent-based species tree construction based on the 481-orthogroup dataset, yielding a topology identical to topology III with a bootstrap value increasing from 50 to 54\% (Supplementary Fig. $17 b)$. Furthermore, we performed a phylogenetic analysis on the basis of a concatenated sequence alignment of 78 chloroplast genes, yielding a topology consistent with topology III with strong bootstrap support (Supplementary Fig. 18). To continue our investigation, we identified both eudicot- and monocot-specific gene families present in the Liriodendron genome based on the PLAZA 3.0 Monocots database (Supplementary Fig. 19). The gene families from either clade were not significantly over-represented in Liriodendron compared with Amborella $\left(\chi^{2}=0.1166 ; P=0.7328\right)$, whereas a monocot plant and a eudicot plant both showed significant biases towards their respective gene families (Fig. 2c). Overall, considering our results, including the mosaic phenotypic characterization, individual and multiple gene tree reconstructions, and lineage-specific gene family identification, we suggest a topology in which eudicots and monocots form a clade that is sister to magnoliids, represented by Liriodendron, with the basal angiosperm Amborella being the next group (Fig. 2d); that is, magnoliids arose before the divergence of eudicots and monocots. Thus, the phylogenetic analysis incorporating the Liriodendron genome provides additional insights into the systematic position and evolution of magnoliids.

At present, the Liriodendron genus contains only two species in regions with a humid subtropical climate, and has partially expanded to the southern margin of the warm temperate climate zone of the Northern Hemisphere ${ }^{21,22}$ (Fig. 3a and 


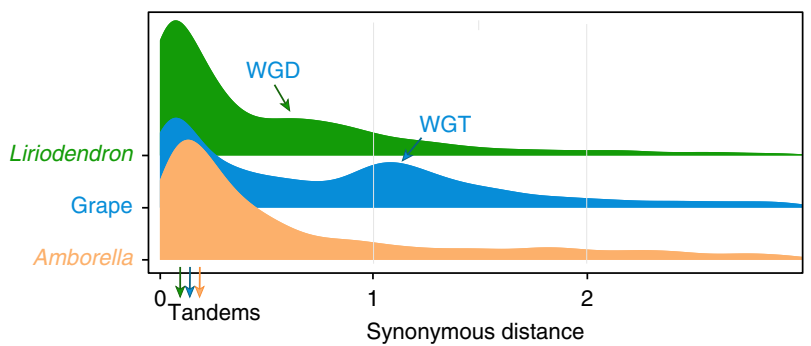

b
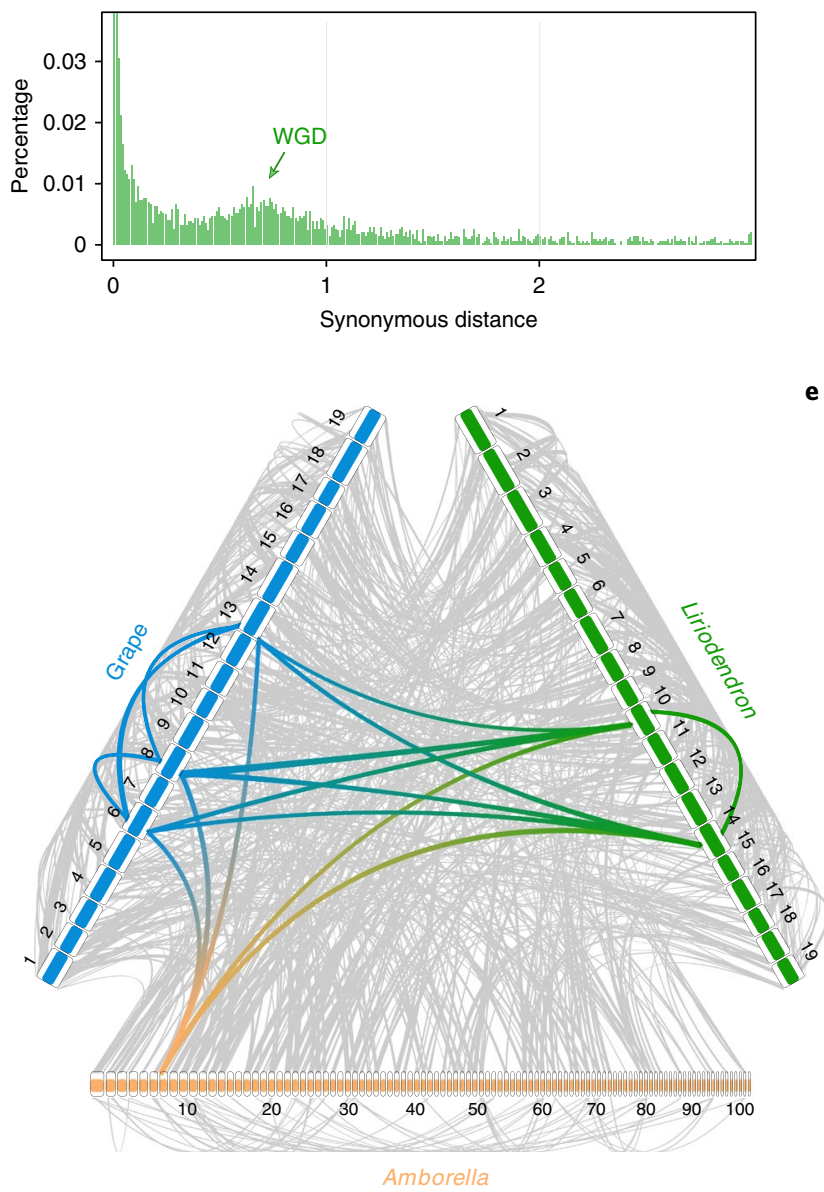

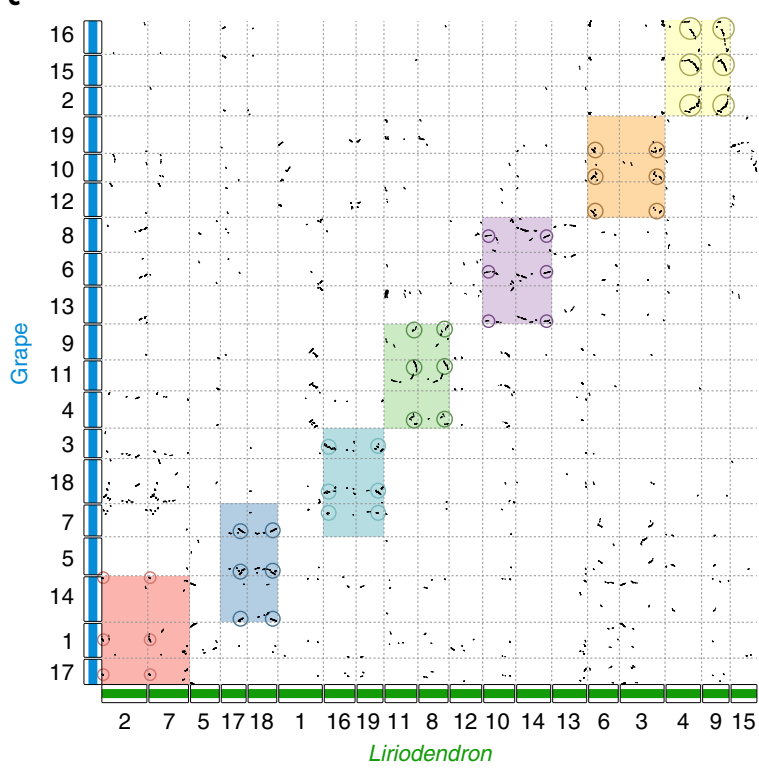

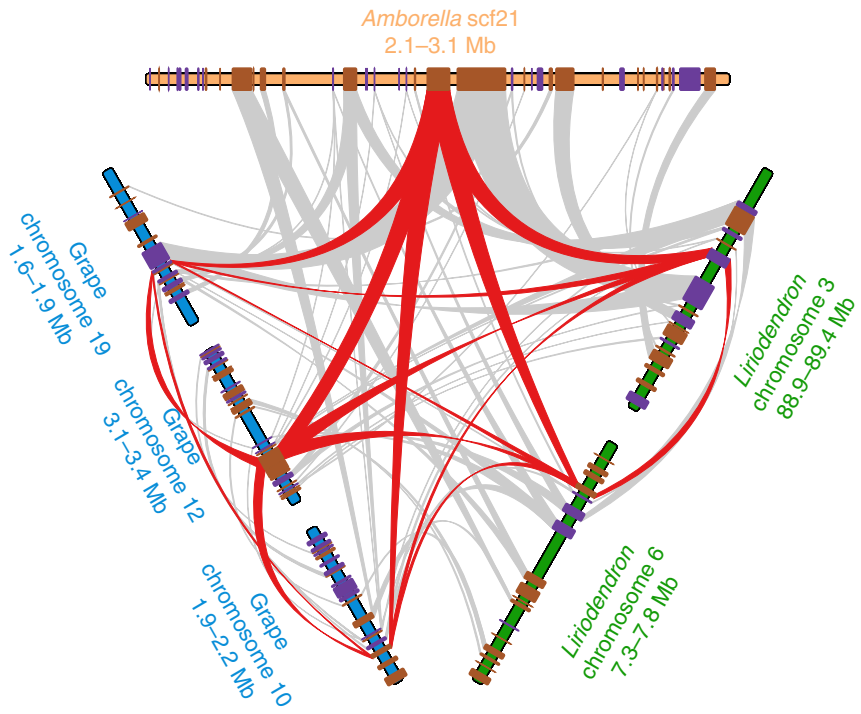

Fig. 1 | Liriodendron lineage-specific WGD. $\mathbf{a}, K_{\mathrm{s}}$ distributions for the whole paranome identified from the whole genome of Liriodendron (green), grape (blue) and Amborella (orange). WGT, whole-genome triplication. $\mathbf{b}, K_{\mathrm{s}}$ distribution for the whole paranome identified from the whole transcriptome of L. chinense. c, Comparison of Liriodendron and grape genomes. Dot plots of orthologues show a 2-3 chromosomal relationship between the Liriodendron genome and grape genome. d, Macrosynteny patterns show that a typical ancestral region in the basal angiosperm Amborella can be tracked to up to two regions in Liriodendron and to up to three regions in the grape. Grey wedges in the background highlight major syntenic blocks spanning more than 30 genes between the genomes (highlighted by one syntenic set shown in colour). e. Microcollinearity patterns between genomic regions from Amborella, Liriodendron and the grape. Rectangles represent predicted gene models, with purple and brown showing relative gene orientations. Grey wedges connect matching gene pairs, with two sets highlighted in red.

Supplementary Fig. 20). However, a number of extinct Liriodendron species were once widely distributed in relatively high-latitude regions of the Northern Hemisphere before a general cooling of the climate occurred during in the Late Tertiary ${ }^{23}$, based on fossil records of seeds and leaves (Fig. 3a and Supplementary Fig. 21). To explore the historical demographic fluctuations and presentday genetic diversity within these two Liriodendron species, we resequenced 20 Liriodendron accessions, including 14 L. chinense individuals and six L. tulipifera individuals (Fig. 3a, Supplementary Fig. 22 and Supplementary Table 15).
On the basis of phylogenetic analysis of a whole-genome single nucleotide polymorphism (SNP) analysis, we found that these Liriodendron accessions formed three distinct phylogenetic groups (Fig. 3b and Supplementary Fig. 23). This was further supported by a principal component analysis (Fig. $3 \mathrm{c}$ ) and structure analysis (Supplementary Fig. 24). All L. chinense individuals from western China (CW) clustered together, and the rest of the L. chinense, collected from eastern China (CE), clustered into the second group. The third group comprised all L. tulipifera individuals collected from North America (NA). It is evident that the NA group is 


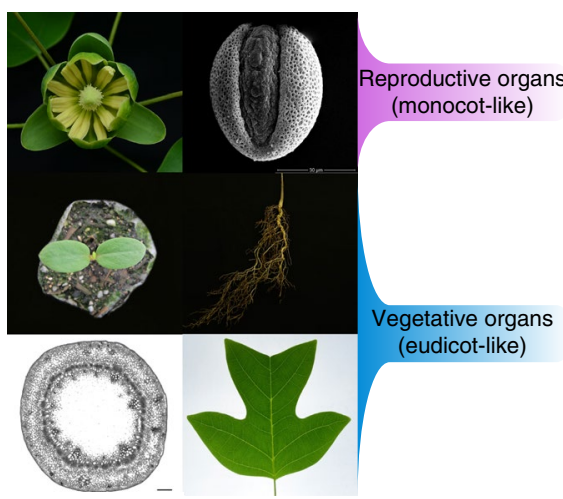

C

\begin{tabular}{|c|c|c|}
\hline & $\begin{array}{c}\text { Eudicot-specific } \\
\text { gene families } \\
(114)\end{array}$ & $\begin{array}{c}\text { Monocot-specific } \\
\text { gene families } \\
(93)\end{array}$ \\
\hline A. trichopoda & 29 & 16 \\
\hline chinense & 52 & 31 \\
\hline S. polyrhiza & 15 & 25 \\
\hline M. cordata & 78 & 19 \\
\hline 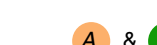 & $\chi^{2}$ & 0.1166 \\
\hline$\underset{\Phi}{\Phi}$ & $P$ & 0.7328 \\
\hline ర్ర & $\chi^{2}$ & 15.6910 \\
\hline ত্ & $P$ & 0.0004 \\
\hline$\frac{1}{c}$ & $\chi^{2}$ & 10.7940 \\
\hline & $P$ & 0.0010 \\
\hline
\end{tabular}

b

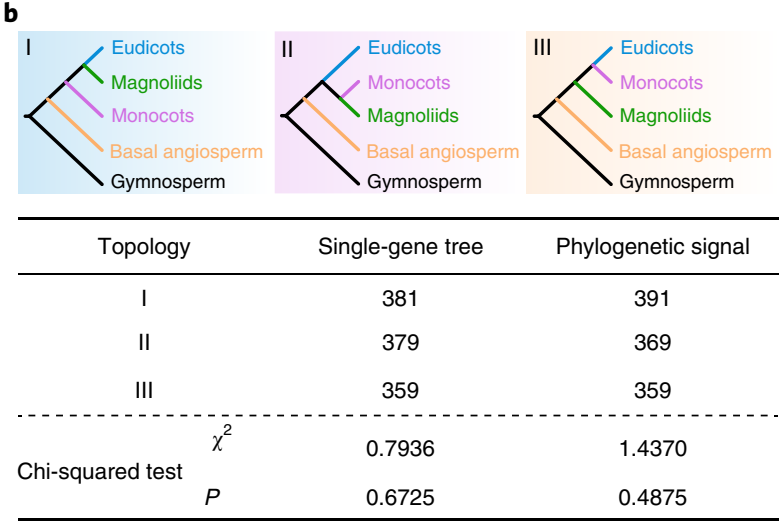

d

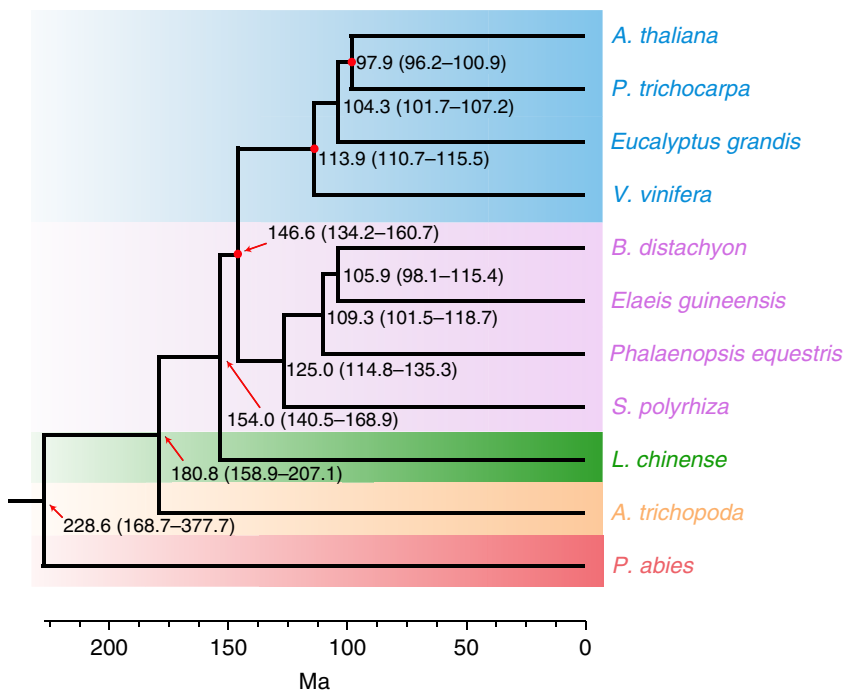

Fig. 2 | Phylogenetic relationships among magnoliids, eudicots and monocots. a, Liriodendron shows typical features of monocots in its reproductive organs (flower parts in multiples of three and monosulcate pollen grains) and of eudicots in its vegetative organs (two cotyledons, a taproot system, a eudicot-like stem cross-section and netted venation). These experiments were repeated independently at least ten times with similar results. Scale bar, $200 \mu \mathrm{m}$. b. Three topologies that coincided with three alternative phylogenetic hypotheses are plotted, and the results of a chi-squared test of the orthogroup numbers supporting each topology are shown below, revealing no statistically significant difference in topology preference. c, The eudicot- and monocot-specific gene families present in Liriodendron are statistically similar to those present in Amborella, whereas Spirodela polyrhiza has a bias towards monocot-specific gene families, and Macleaya cordata has a bias towards eudicot-specific gene families when compared with Amborella. d, Dated phylogeny for 11 plant species with Picea abies as an outgroup. A time scale is shown at the bottom, and red points in some nodes indicate fossil calibration points.

phylogenetically positioned between the two L. chinense groups and more closely related to the CE group, suggesting that the earliest divergence occurred between the populations in eastern China and those in western China, followed by differentiation between the eastern Chinese populations and North American populations. This pattern is supported by the phenotypic analysis, which shows that all three groups share one leaf morphological feature, while the CE and NA groups have their own unique leaf morphological feature (Supplementary Fig. 25). Fossil records indicate that similar leaf morphological features to those in the western and eastern China groups had already emerged in two extinct Liriodendron species $^{24,25}$, again suggesting that these two L. chinense groups may have diverged a very long time ago, possibly preceding the intercontinental differentiation between $L$. chinense and L. tulipifera (Supplementary Fig. 25).

Nucleotide diversity $(\pi)$ analysis shows that the CW group has the highest genetic diversity, followed by the CE group, and that the genetic diversity of the NA group is tenfold lower than that of the CW group (Fig. 3d). An analysis of demographic history using the pairwise sequentially Markovian coalescent (PSMC) model $^{26}$ shows that the two groups from China both had population size peaks at approximately $0.4 \mathrm{Ma}$ and declined afterwards, whereas the NA group population size peak occurred much earlier and continuously declined since approximately 2.3 Ma (Fig. 4), indicating that the populations in eastern China and those in western China underwent a similar demographic history different from that in North American populations. We also calculated genetic differentiation statistics (fixation index; $F_{\mathrm{ST}}$ ) among the three Liriodendron groups, indicating that the genetic differentiation $\left(F_{\mathrm{ST}}=0.2055\right)$ between the NA group and the CW group was slightly lower than that $\left(F_{\mathrm{ST}}=0.2707\right)$ between the NA group and the CE group (Fig. $\left.3 \mathrm{~d}\right)$. In addition, we also found that the $\mathrm{CW}$ group had the highest level of individual differences compared with the other two geographical groups (Supplementary Fig. 26).

The natural distribution areas of these two Liriodendron species on their respective continents are highly consistent with the two principal areas where Tertiary relict floras occur ${ }^{23}$ (Fig. 3a). Although Liriodendron species were once distributed over the 
a
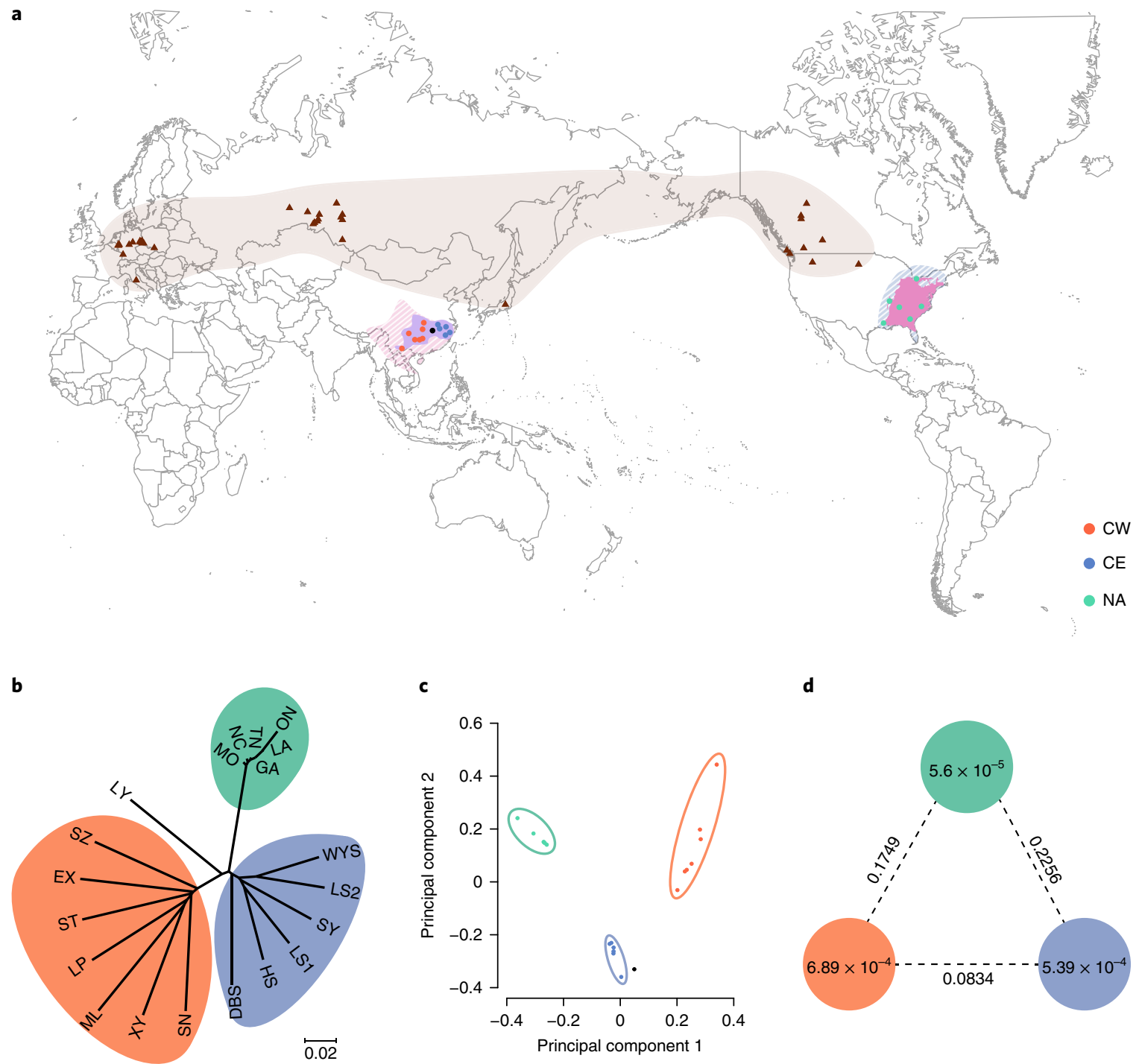

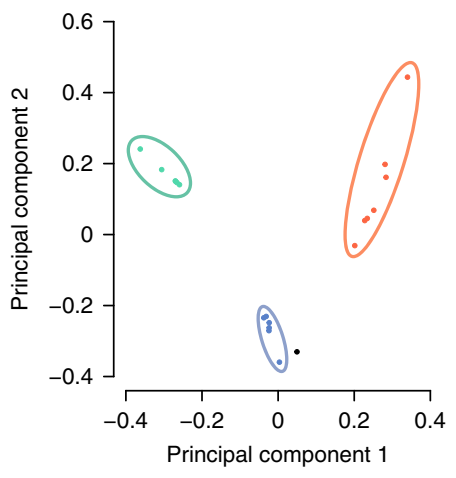

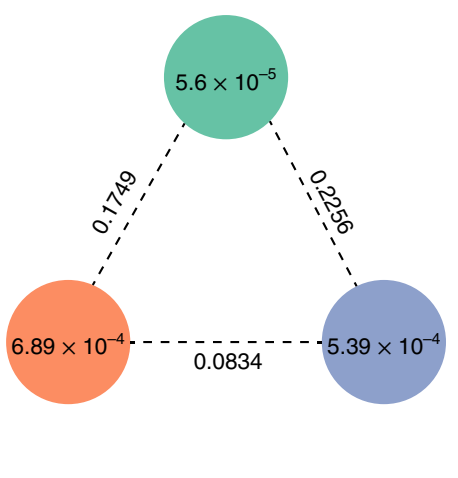

Fig. 3 | Geographic distribution and population diversity of Liriodendron accessions. a, Geographic distribution of Liriodendron accessions. Brown triangles represent the fossil distribution of Liriodendron plants in high-latitude regions of the Northern Hemisphere. Fringe patterns show two principal refugia where Tertiary relict floras occurred: southern East Asia and eastern North America. The natural distributions of $L$. chinense and $L$. tulipifera are plotted, with coloured dots representing individual Liriodendron accessions. b, Neighbour-joining tree of all accessions constructed from whole-genome SNPs. Accessions coming from the same geographic areas are grouped together and coloured corresponding to the colours used in a. LY, Liu Yang; SZ, Sang Zhi; EX, E Xi; ST, Song Tao; LP, Li Ping; ML, Meng La; XY, Xu Yong; SN, Sui Ning; DBS, DaBie Shan; HS, Huang Shan; LS1, Lushan_1; SY, Song Yang; LS2, Lushan_2; WYS, WuYi Shan; ON, Ontario; LA, Louisiana; GA, Georgia; TN, Tennessee; NC, North Carolina; MO, Missouri. c, Principal component analysis plots of the first two components for all 20 accessions, with dots coloured corresponding to their provenances. d, Nucleotide diversity $(\pi)$ and population divergence $\left(F_{S T}\right)$ across the three groups. The value in each circle represents a measure of nucleotide diversity for this group, and the value on each line indicates the population divergence between the two groups.

high-latitude regions of Europe (Fig. 3a), the east-west-orientated mountains are thought to have blocked their southward migration during global cooling in the Late Tertiary and subsequent Quaternary glaciations ${ }^{27}$, finally leading to the extinction of Liriodendron in Europe ${ }^{7}$. With respect to the Liriodendron that survived in East Asia and eastern North America, the higher genetic diversity of $L$. chinense compared with $L$. tulipifera is consistent with the greater number of suitable refugia in East Asia ${ }^{28,29}$. In this study, we observed a sustained population decrease during the whole Quaternary glaciation in all L. tulipifera accessions and a population recovery approximately $0.3-0.4 \mathrm{Ma}$ in all L. chinense accessions (Fig. 4), which may have contributed considerably to the severe loss of genetic diversity in L. tulipifera and the relatively high retention of genetic diversity in L. chinense (Fig. 3d and Supplementary Fig. 27), respectively. The population recovery observed in all $L$. chinense accessions occurred in the interglacial stage between the Guxiang Glaciation (0.3-0.13 Ma) and Naynayxungla Glaciation (0.72$0.5 \mathrm{Ma})^{30}$. Considering that the Naynayxungla Glaciation was the most extensive glaciation, including large ice caps and massive valley glaciers, and the following Guxiang Glaciation was characterized by valley glaciers only ${ }^{30}$, we speculate that the temperature recovery and deglaciation during this interglacial stage provided a foundation for L. chinense population recovery within East Asian refugia. Consequently, in addition to the higher habitat diversity within East Asian refugia ${ }^{29}$, a suitable living environment during the interglacial stage between the Naynayxungla and Guxiang glaciations may 

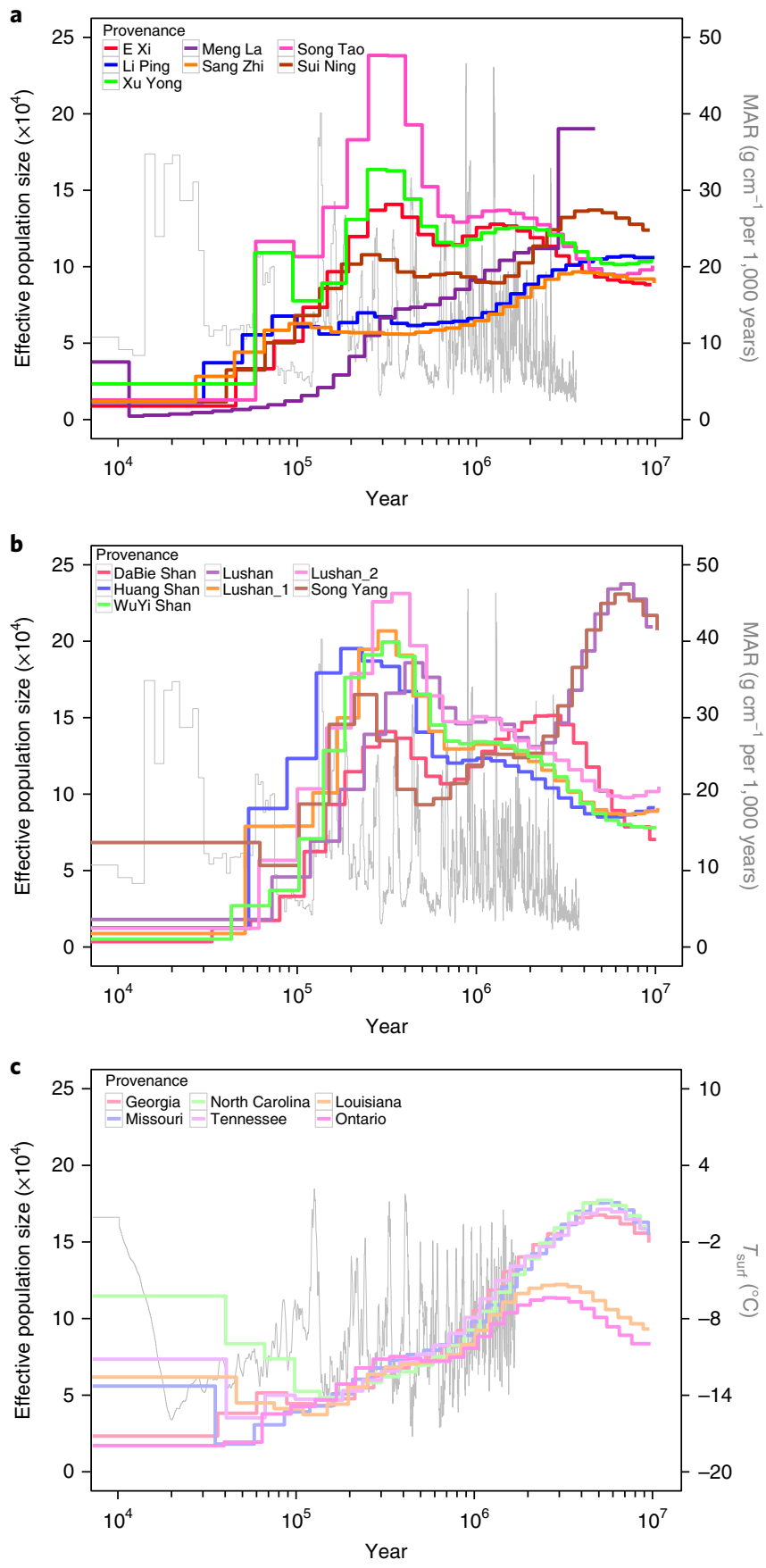

Fig. 4 | Historical fluctuations in effective population size. a-c, Plots of PSMC results for 20 individuals ( 7 from western China (a); 7 from eastern China (b); and 6 from North America (c)), as indicated in each legend. The grey lines represent the mass accumulation rate (MAR) of the Chinese Loess Plateau in $\mathbf{a}$ and $\mathbf{b}$, and the atmospheric surface air temperature relative to the present in $\mathbf{c}$.

have contributed to the retention, restoration and augmentation of L. chinense genetic diversity.

\section{Methods}

Plant materials and sequencing. For genome sequencing, we collected fresh leaves from an adult plant of $L$. chinense grown in Lushan, which is located in the Jiangxi province of China. For Illumina sequencing, four series of paired-end sequencing libraries with insert sizes of 170, 250, 500 and 800 bp were constructed and subsequently sequenced on the Illumina HiSeq 2000 platform, ultimately resulting in $327.11 \mathrm{~Gb}$ clean reads. For PacBio single-molecule real-time sequencing, sequencing libraries with 20-kb DNA inserts were constructed and subsequently sequenced on the Pacific Biosciences RSII instrument, ultimately resulting in a total of $150.18 \mathrm{~Gb}$ subread with an N50 length of $15.96 \mathrm{~kb}$ for the genome assembly. In addition, purified DNA was labelled at Nt.BspQI sites using the IrysPrep kit, and a $315.41 \mathrm{~Gb}$ optical map of the sample was produced from the BioNano Irys system. In addition, abundances of 17-nucleotide k-mers from 170- and 250-bp Illumina sequencing libraries were used to estimate the genome size.

De novo assembly. The Liriodendron genome was de novo assembled using FALCON (https://github.com/PacificBiosciences/FALCON) based on PacBio long reads (only reads longer than $10 \mathrm{~kb}$ were used in the assembly). Errors in the PacBio reads were corrected within the FALCON pipeline. Contigs was first polished based on raw PacBio data and finally corrected using Illumina short reads with Pilon ${ }^{31}$. A hybrid assembly was created based on contigs and optical maps using the Bionano Solve Pipeline (https://bionanogenomics.com/supportpage/bionano-access/). Then, the corrected PacBio long reads were used for superscaffold gap filling using PBJelly ${ }^{32}$. We constructed a reference genetic map of L. chinense based on an $\mathrm{F}_{1}$ population of 150 plants from a cross between L. chinense and L. tulipifera using JoinMap 4.0 (ref. ${ }^{33}$ ). Markers with inconsistent placement were manually screened and the collinearity of common markers was inspected using MapChart 2.2 (ref. ${ }^{33}$ ). Markers in common were used as anchor points. Possible chimeric scaffolds were identified as those containing sequences of markers mapped to different locations in the same linkage group or different linkage groups, and these scaffolds were manually inspected. This process generated 19 Liriodendron pseudomolecules.

Genome assessment. We assessed the coverage of the genome assembly by mapping 89 BACs back to assembly with $97 \%$ of these BAC sequences covered without any obvious misassemblies. A comparison of 9 randomly chosen BACs sequenced by 454 sequencing technology indicated a low error rate. In addition, we used the $\mathrm{BUSCO}^{34}$ database to assess the genome assembly. We also validated the assembled genome using 66,934 unigenes (length $\geq 200 \mathrm{bp}$ ) from RNA-Seq.

Repeat annotation. We identified tandem repeats and transposable elements separately. Tandem repeats were predicted using Tandem Repeats Finder 4.04 (ref. ${ }^{35}$ ). For transposable element identification, we performed a combination of similarity-based and de novo approaches. First, we used RepeatMasker with the Repbase 16.10 (ref. ${ }^{36}$ ) database of known repeat sequences to search for transposable elements in the genome, and we additionally used RepeatProteinMask, implemented in RepeatMasker, to identify transposable elements by aligning the genome sequence to the transposable element protein database. Then, to apply our de novo approach, we constructed a repeat library generated by RepeatModeler ${ }^{37}$ with default parameters and ran RepeatMasker on the genome sequences, using the RepeatModeler consensus sequence as a library. Finally, all the repeat sequences identified by the different methods were combined into the final repeat annotation.

Gene prediction. Gene model prediction was conducted by the MAKER pipeline ${ }^{38}$, integrating ab initio prediction with de novo assembled transcripts from shortread messenger RNA sequencing, isoform-sequencing full-length transcripts, and protein homology data. A high-confidence gene model was constructed by further removing transposons and low-confidence predictions. Gene functional annotation was performed using the Swiss-Prot and TrEMBL databases ${ }^{39}$, while motifs and domains were annotated using InterProScan ${ }^{40}$ by searching against publicly available protein databases. Descriptions of gene products (that is, Gene Ontology terms) were retrieved from the corresponding InterPro entries. We also mapped the Liriodendron reference genes to $\mathrm{KEGG}^{41}$ pathway maps.

Transfer RNA genes were predicted based on tRNAscan-SE ${ }^{42}$. Ribosomal RNA fragments were identified by aligning plant ribosomal RNA sequences ${ }^{43}$ to the Liriodendron genome by BLASTN ${ }^{44}$. micro RNA and small nuclear RNA genes were detected by INFERNAL ${ }^{45}$ software against the Rfam database ${ }^{46}$ (release 9.1).

Genome synteny. We performed synteny searches to compare the $L$. chinense genome structure with that of the grape and Amborella genomes using MCscan ${ }^{47}$, requiring at least five gene pairs per syntenic block. The resulting dot plots were inspected to confirm the paleoploidy level of $L$. chinense in relation to the other genomes by counting the syntenic depth in each genomic region.

$K_{\mathrm{s}}$ values for homologous gene pairs were calculated as described in Maere et al. ${ }^{48}$. Fourfold synonymous third-codon transversion position values were calculated for syntenic segments from the concatenated alignments and constructed by dividing the number of transversions at all fourfold degenerate third-codon positions by the number of fourfold degenerate third-codon positions.

Phylogenetic analysis. Orthogroups were constructed with 14 other sequenced plants -6 eudicots (Arabidopsis thaliana, Populus trichocarpa, Vitis vinifera, Coffea canephora, Ipomoea nil and Fraxinus excelsior); 6 monocots (Brachypodium distachyon, Xerophyta viscosa, Asparagus officinalis, Musa acuminata, Ananas comosus and Oryza sativa); 1 basal angiosperm (Amborella trichopoda); and 1 gymnosperm (Gnetum montanum) - and three other magnoliid transcriptome 
datasets, including two sequenced in this study (Magnolia grandiflora and Michelia alba) and one available in Ibarra-Laclette et al..$^{49}$ (Persea americana), using the software OrthoFinder ${ }^{50}$. We selected low-copy orthogroups with the number of putative orthologues less than two in each species, and putative orthologues were found in at least four eudicots, four monocots, three magnoliids, one basal angiosperm and one gymnosperm, resulting in 1,163 orthogroups. Then, each orthogroup was aligned using Clustal Omega ${ }^{51}$, and all alignments were further trimmed using TrimAl 1.2 (ref. ${ }^{52}$ ). Next, we constructed 1,163 single-gene trees using RAxML ${ }^{53}$ with the PROTCATWAG mode. Then, we compared these single-gene trees with the species tree and screened them as described in Zeng et al. ${ }^{6}$ Finally, after careful examination, a total of 502 low-copy orthogroups were selected for further analysis.

We also calculated the phylogenetic signal based on three alternative topological hypotheses and quantified the difference in gene-wise log-likelihood scores ( $\Delta$ GLS) among each of the three topologies using RAxML ${ }^{20,53}$. To diminish the influence of tiny amounts of data on phylogenetic inference, we further excluded orthogroups with outlier $\Delta$ GLS values, defined as described in Shen et al. ${ }^{20}$. To estimate the species tree, we performed a coalescent-based approach using Astral 5.6.1 (ref. ${ }^{54}$ ). We also performed phylogenetic analyses based on 78 chloroplast genes among 24 land plant species using RAxML ${ }^{53}$.

To estimate divergence time, we used PAML MCMCTREE ${ }^{55}$ to perform Bayesian estimation with soft fossil constraints ${ }^{56}$ based on 235 single-copy orthologous genes that are shared by L. chinense and 10 other species. Markov chain Monte Carlo analysis was run to sample 1,000,000 times with a sampling frequency of 50 and a burn-in of 5,000,000 iterations. We also used $\mathrm{CAFE}^{57}$ to identify gene families that had undergone expansions or contractions across the maximum likelihood tree.

Resequencing and diversity analysis. DNA from $14 \mathrm{~L}$. chinense and 6 L. tulipifera adult plants was extracted, and paired-end libraries with insert sizes of 100-150 bp were sequenced using Illumina technology at BGI. We first called SNPs using BWA $^{58}$, GATK $^{59}$ and SAMtools ${ }^{60}$, then annotated these SNPs using SNPEFF ${ }^{61}$, ultimately summarizing them by a customized Perl script.

The neighbour-joining phylogenetic tree was constructed using TreeBeST ${ }^{62}$ based on SNPs. Population structure and ancestry information was inferred using FRAPPE ${ }^{63}$ with the best $K$ value determined by ADMIXTURE $^{64}$ based on a crossvalidation test. We additionally performed a principal component analysis using the STRATPCA programme from EIGENSOFT 3.2 (ref. ${ }^{65}$ ).

Population genetic parameters, including nucleotide diversity $(\pi)^{66}$ and the Watterson estimator $\left(\theta_{\mathrm{w}}\right)^{67}$, were estimated on the basis of the genotypes of each line at the SNP positions using BioPerl.

The PSMC model, which was originally applied to human genomes ${ }^{26}$ and subsequently also applied to plant genomes ${ }^{15,68}$, was applied to study the effective population sizes $\left(N_{\mathrm{e}}\right)$ of the two Liriodendron species over time.

See the Supplementary Note for additional details.

Reporting Summary. Further information on research design is available in the Nature Research Reporting Summary linked to this article.

Code availability. The custom Perl script used for summarization of SNP annotation is available from the corresponding author upon request.

\section{Data availability}

The raw reads and genome assembly have been deposited as a BioProject under accession PRJNA418360. The resequencing data for 20 Liriodendron individuals have been deposited as a BioProject under accession PRJNA418361.

Received: 30 November 2017; Accepted: 8 November 2018; Published online: 17 December 2018

\section{References}

1. Soltis, P. S. \& Soltis, D. E. The origin and diversification of angiosperms. Am. J. Bot. 91, 1614-1626 (2004).

2. Qui, Y. L. et al. Phylogenetic analyses of basal angiosperms based on nine plastid, mitochondrial, and nuclear genes. Int. J. Plant Sci. 166, 815-842 (2005).

3. Moore, M. J. \& Soltis, D. E. Phylogenetic analysis of the plastid inverted repeat for 244 species: insights into deeper-level angiosperm relationships from a long, slowly evolving sequence region. Int. J. Plant Sci. 172, 541-558 (2011).

4. Zhang, N., Zeng, L., Shan, H. \& Ma, H. Highly conserved low-copy nuclear genes as effective markers for phylogenetic analyses in angiosperms. New Phytol. 195, 923-937 (2012).

5. Wickett, N. J. et al. Phylotranscriptomic analysis of the origin and early diversification of land plants. Proc. Natl Acad. Sci. USA 111, E4859-E4868 (2014)

6. Zeng, L. et al. Resolution of deep angiosperm phylogeny using conserved nuclear genes and estimates of early divergence times. Nat. Commun. 5, 4956 (2014).
7. Parks, C. R. \& Wendel, J. F. Molecular divergence between Asian and North American species of Liriodendron (Magnoliaceae) with implications for interpretation of fossil floras. Am. J. Bot. 77, 1243-1256 (1990).

8. Azuma, H., Garcia-Franco, J. G., Rico-Gray, V. \& Thien, L. B. Molecular phylogeny of the Magnoliaceae: the biogeography of tropical and temperate disjunctions. Am. J. Bot. 88, 2275-2285 (2001).

9. Nie, Z. L. et al. Phylogenetic and biogeographic complexity of Magnoliaceae in the Northern Hemisphere inferred from three nuclear data sets. $\mathrm{Mol}$. Phylogenet. Evol. 48, 1027-1040 (2008).

10. Parks, C. R., Miller, N. G., Wendel, J. F. \& McDougal, K. M. Genetic divergence within the genus Liriodendron (Magnoliaceae). Ann. MO Bot. Gard. 70, 658-666 (1983).

11. Darwin, C. R. On the Origin of Species by Means of Natural Selection or the Preservation of Favoured Races in the Struggle for Life (John Murray, London, 1859).

12. Coyne, J. A. Why Evolution Is True (Univ. Press, Oxford, 2009).

13. Wendel, J. F., Jackson, S. A., Meyers, B. C. \& Wing, R. A. Evolution of plant genome architecture. Genome Biol. 17, 37 (2016).

14. Jaillon, O. et al. The grapevine genome sequence suggests ancestral hexaploidization in major angiosperm phyla. Nature 449, 463-467 (2007).

15. Amborella Genome, P. The Amborella genome and the evolution of flowering plants. Science 342, 1241089 (2013).

16. Cui, L. et al. Widespread genome duplications throughout the history of flowering plants. Genome Res. 16, 738-749 (2006).

17. Kumar, S., Stecher, G., Suleski, M. \& Hedges, S. B. TimeTree: a resource for timelines, timetrees, and divergence times. Mol. Biol. Evol. 34, 1812-1819 (2017)

18. Hollister, J. D. \& Gaut, B. S. Epigenetic silencing of transposable elements: a trade-off between reduced transposition and deleterious effects on neighboring gene expression. Genome Res. 19, 1419-1428 (2009).

19. Rose, A. B. \& Beliakoff, J. A. Intron-mediated enhancement of gene expression independent of unique intron sequences and splicing. Plant Physiol. 122, 535-542 (2000).

20. Shen, X. X., Hittinger, C. T. \& Rokas, A. Contentious relationships in phylogenomic studies can be driven by a handful of genes. Nat. Ecol. Evol. 1, 126 (2017).

21. Little, E. L. Atlas of United States Trees. Volume 1: Conifers and Important Hardwoods (US Department of Agriculture, 1971).

22. Hao, R. M. Geographical distribution of Liriodendron chinense in China and its significance. J. Plant Resour. Environ. (China) 4, 1-6 (1995).

23. Milne, R. I. Northern Hemisphere plant disjunctions: a window on tertiary land bridges and climate change? Ann. Bot. 98, 465-472 (2006)

24. Baghai, N. L. Liriodendron (Magnoliaceae) from the Miocene Clarkia flora of Idaho. Am. J. Bot. 75, 451-464 (1988).

25. Bell, W. A. Upper Cretaceous floras of the Dunvegan, Bad Heart, and Milk River Formations of Western Canada. Geol. Surv. Canada Bull. 94 1-76 (1962).

26. Li, H. \& Durbin, R. Inference of human population history from individual whole-genome sequences. Nature 475, 493-496 (2011).

27. Tiffney, B. H. The Eocene North Atlantic land bridge: its importance in Tertiary and modern phytogeography of the Northern Hemisphere. J. Arnold Arbor. 66, 243-273 (1985).

28. Wen, J. Evolution of eastern Asian and eastern North American disjunct distributions in flowering plants. Annu. Rev. Ecol. Syst. 30, 421-455 (1999).

29. Qian, H. \& Ricklefs, R. E. Large-scale processes and the Asian bias in species diversity of temperate plants. Nature 407, 180-182 (2000).

30. Sun, Y. B. \& An, Z. S. Late Pliocene-Pleistocene changes in mass accumulation rates of eolian deposits on the central Chinese Loess Plateau. J. Geophys. Res. Atmos. 110, D23101 (2005).

31. Walker, B. J. et al. Pilon: an integrated tool for comprehensive microbial variant detection and genome assembly improvement. PLOS ONE 9 , e112963 (2014).

32. English, A. C. et al. Mind the gap: upgrading genomes with Pacific Biosciences RS long-read sequencing technology. PLoS ONE 7, e47768 (2012)

33. Van Ooijen, J. W. JoinMap 4: Software for the Calculation of Genetic Linkage Maps in Experimental Populations (Kyazma B.V., Wageningen, 2006).

34. Simao, F. A., Waterhouse, R. M., Ioannidis, P., Kriventseva, E. V. \& Zdobnov, E. M. BUSCO: assessing genome assembly and annotation completeness with single-copy orthologs. Bioinformatics 31, 3210-3212 (2015).

35. Benson, G. Tandem repeats finder: a program to analyze DNA sequences. Nucleic Acids Res. 27, 573-580 (1999).

36. Jurka, J. et al. Repbase Update, a database of eukaryotic repetitive elements. Cytogenet. Genome Res. 110, 462-467 (2005).

37. Price, A. L., Jones, N. C. \& Pevzner, P. A. De novo identification of repeat families in large genomes. Bioinformatics 21, i351-i358 (2005).

38. Cantarel, B. L. et al. Maker: an easy-to-use annotation pipeline designed for emerging model organism genomes. Genome Res. 18, 188-196 (2008).

39. Bairoch, A. \& Apweiler, R. The Swiss-Prot protein sequence database and its supplement trembl in 2000. Nucleic Acids Res. 28, 45-48 (2000). 
40. Zdobnov, E. M. \& Apweiler, R. InterProScan-an integration platform for the signature-recognition methods in InterPro. Bioinformatics 17, 847-848 (2001).

41. Kanehisa, M. \& Goto, S. KEGG: kyoto encyclopedia of genes and genomes. Nucleic Acids Res. 28, 27-30 (2000).

42. Lowe, T. M. \& Eddy, S. R. tRNAscan-SE: a program for improved detection of transfer RNA genes in genomic sequence. Nucleic Acids Res. 25, 955-964 (1997).

43. Garcia, S., Galvez, F., Gras, A., Kovarik, A. \& Garnatje, T. Plant rDNA database: update and new features. Database 2014, 1-7 (2014).

44. Altschul, S. F., Gish, W., Miller, W., Myers, E. W. \& Lipman, D. J. Basic local alignment search tool. J. Mol. Biol. 215, 403-410 (1990).

45. Nawrocki, E. P., Kolbe, D. L. \& Eddy, S. R. Infernal 1.0: inference of RNA alignments. Bioinformatics 25, 1335-1337 (2009)

46. Griffithsjones, S. et al. Rfam: annotating non-coding RNAs in complete genomes. Nucleic Acids Res. 33, 121-124 (2005).

47. Wang, Y. et al. MCScanX: a toolkit for detection and evolutionary analysis of gene synteny and collinearity. Nucleic Acids Res. 40, e49 (2012)

48. Maere, S. et al. Modeling gene and genome duplications in eukaryotes. Proc. Natl Acad. Sci. USA 102, 5454-5459 (2005).

49. Ibarra-Laclette, E. et al. Deep sequencing of the Mexican avocado transcriptome, an ancient angiosperm with a high content of fatty acids BMC Genom. 16, 599 (2015).

50. Emms, D. M. \& Kelly, S. OrthoFinder: solving fundamental biases in whole genome comparisons dramatically improves orthogroup inference accuracy. Genome Biol. 16, 157 (2015).

51. Sievers, F. \& Higgins, D. G. Clustal Omega for making accurate alignments of many protein sequences. Protein Sci. 27, 135-145 (2018).

52. Capella-Gutierrez, S., Silla-Martinez, J. M. \& Gabaldon, T. TrimAl: a tool for automated alignment trimming in large-scale phylogenetic analyses. Bioinformatics 25, 1972-1973 (2009).

53. Stamatakis, A. RAxML-VI-HPC: maximum likelihood-based phylogenetic analyses with thousands of taxa and mixed models. Bioinformatics $\mathbf{2 2}$ 2688-2690 (2006).

54. Mirarab, S. et al. ASTRAL: genome-scale coalescent-based species tree estimation. Bioinformatics 30, i541-i548 (2014).

55. Yang, Z. PAML 4: phylogenetic analysis by maximum likelihood. Mol. Biol. Evol. 24, 1586-1591 (2007)

56. Yang, Z. \& Rannala, B. Bayesian estimation of species divergence times under a molecular clock using multiple fossil calibrations with soft bounds. Mol. Biol. Evol. 23, 212-226 (2006).

57. De Bie, T., Cristianini, N., Demuth, J. P. \& Hahn, M. W. CAFE: a computational tool for the study of gene family evolution. Bioinformatics 22 1269-1271 (2006).

58. Li, H. \& Durbin, R. Fast and accurate short read alignment with BurrowsWheeler transform. Bioinformatics 25, 1754-1760 (2009).

59. DePristo, M. A. et al. A framework for variation discovery and genotyping using next-generation DNA sequencing data. Nat. Genet. 43, 491-498 (2011).

60. Li, H. et al. The Sequence Alignment/Map format and SAMtools. Bioinformatics 25, 2078-2079 (2009).

61. Cingolani, P. et al. A program for annotating and predicting the effects of single nucleotide polymorphisms, SnpEff: SNPs in the genome of Drosophila melanogaster strain w1118; iso-2; iso-3. Fly (Austin) 6, 80-92 (2012).

62. Vilella, A. J. et al. EnsemblCompara GeneTrees: complete, duplication-aware phylogenetic trees in vertebrates. Genome Res. 19, 327-335 (2009).

63. Tang, H., Peng, J., Wang, P. \& Risch, N. J. Estimation of individual admixture: analytical and study design considerations. Genet. Epidemiol. 28, 289-301 (2005).

64. Alexander, D. H., Novembre, J. \& Lange, K. Fast model-based estimation of ancestry in unrelated individuals. Genome Res. 19 1655-1664 (2009).
65. Price, A. L. et al. Principal components analysis corrects for stratification in genome-wide association studies. Nat. Genet. 38, 904-909 (2006).

66. Nei, M. \& Li, W. H. Mathematical model for studying genetic variation in terms of restriction endonucleases. Proc. Natl Acad. Sci. USA 76, 5269-5273 (1979).

67. Watterson, G. A. On the number of segregating sites in genetical models without recombination. Theor. Popul. Biol. 7, 256-276 (1975).

68. Ibarra-Laclette, E. et al. Architecture and evolution of a minute plant genome. Nature 498, 94-98 (2013).

\section{Acknowledgements}

This work was supported by the National High Technology Research and Development Program of China (863 Program; number 2013AA102705), Key Research and Development Plan of Jiangsu Province (BE2017376), Foundation of Jiangsu Forestry Bureau (LYKJ[2017]42), Specialized National Basic Research Program of China (973 Program; 2012CB114504), National Natural Science Foundation of China (31770715), National High-level Personnel of Special Support Program, Qinglan Project of Jiangsu Province, Talent Project by the Ministry of Science and Technology and Priority Academic Program Development of Jiangsu Higher Education Institutions.

\section{Author contributions}

J.S. and J.C. were the leading investigators of this research programme. J.S., J.C., S.Y. and N.L. designed the experiments and coordinated the project. Z.H., P.W., Y.S., Y.Z., Z.W., S.L. and T.C. performed field work and collected samples. X.G., C.Z., P.W, L.Y. and H.X. performed the sequencing experiments. X.G., C.Z., L.X, Qihui Z. and M.S. performed the genome assemblies. Z.H., X.G., C.Z. and L.G. constructed the genetic map. L.X, Q.Z. H.X., X.L. and Y.L. provided RNA-Seq data. X.G., C.Z., L.Y., Q.G. and N.L. performed the gene annotation and genome assembly assessment. J.C., Z.H., X.G., J.Z. and S.Y. performed the genome duplication analysis. Z.H., L.X., L.Y., Y.P. and S.Y. analysed the transposable element insertion. J.C., Z.H., X.G., N.L., S.Y. and J.S. performed the phylogenetic analysis. J.C., Z.H., X.G., T.L., S.Y. and J.S. performed the population genetic structure analysis. J.C., Z.H., S.G., S.Y. and J.S. wrote and edited most of the manuscript. All authors read and approved the final manuscript.

\section{Competing interests}

The authors declare no competing interests.

\section{Additional information}

Supplementary information is available for this paper at https://doi.org/10.1038/ s41477-018-0323-6.

Reprints and permissions information is available at www.nature.com/reprints. Correspondence and requests for materials should be addressed to J.C. or N.L. or S.Y. or J.S.

Publisher's note: Springer Nature remains neutral with regard to jurisdictional claims in published maps and institutional affiliations.

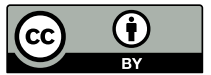

Open Access This article is licensed under a Creative Commons Attribution 4.0 International License, which permits use, sharing, adaptation, distribution and reproduction in any medium or format, as long as you give appropriate credit to the original author(s) and the source, provide a link to the Creative Commons license, and indicate if changes were made. The images or other third party material in this article are included in the article's Creative Commons license, unless indicated otherwise in a credit line to the material. If material is not included in the article's Creative Commons license and your intended use is not permitted by statutory regulation or exceeds the permitted use, you will need to obtain permission directly from the copyright holder. To view a copy of this license, visit http://creativecommons.org/licenses/by/4.0/.

(c) The Author(s) 2018 


\section{natureresearch}

Corresponding author(s): Jisen Shi

\section{Reporting Summary}

Nature Research wishes to improve the reproducibility of the work that we publish. This form provides structure for consistency and transparency in reporting. For further information on Nature Research policies, see Authors \& Referees and the Editorial Policy Checklist.

\section{Statistical parameters}

When statistical analyses are reported, confirm that the following items are present in the relevant location (e.g. figure legend, table legend, main text, or Methods section).

n/a Confirmed

$\bigotimes$ The exact sample size $(n)$ for each experimental group/condition, given as a discrete number and unit of measurement

$\square$ An indication of whether measurements were taken from distinct samples or whether the same sample was measured repeatedly

The statistical test(s) used AND whether they are one- or two-sided

Only common tests should be described solely by name; describe more complex techniques in the Methods section.

Х $\square$ A description of all covariates tested

Х $\square$ A description of any assumptions or corrections, such as tests of normality and adjustment for multiple comparisons

$\triangle \quad$ A full description of the statistics including central tendency (e.g. means) or other basic estimates (e.g. regression coefficient) AND

$X$ variation (e.g. standard deviation) or associated estimates of uncertainty (e.g. confidence intervals)

$\varnothing$ For null hypothesis testing, the test statistic (e.g. $F, t, r$ ) with confidence intervals, effect sizes, degrees of freedom and $P$ value noted

Give P values as exact values whenever suitable.

Х $\square$ For Bayesian analysis, information on the choice of priors and Markov chain Monte Carlo settings

Х $\square$ For hierarchical and complex designs, identification of the appropriate level for tests and full reporting of outcomes

Х $\square$ Estimates of effect sizes (e.g. Cohen's d, Pearson's $r$ ), indicating how they were calculated

$\varnothing$ Clearly defined error bars

State explicitly what error bars represent (e.g. SD, SE, Cl)

Our web collection on statistics for biologists may be useful.

\section{Software and code}

Policy information about availability of computer code

Data collection No software was used to collect the data.

Data analysis We used lots of software for data analysis in this paper. FALCON, SMRT Link v5.0.0, BWA-mem v0.7.17, Pilon v1.21 and PBJelly v15.8.24 were used in genome assembly. JoinMap v4.0 was used in linkage map construction. SOAPdenovo v2.04, BLASTN v2.3.0, Trinity v2.4.0 and BLAT v35 were used in genome assessment. Tandem Repeats Finder v4.04, RepeatMasker, RepeatModeler v1.0.11, TBLASTN v2.3.0, MAKER v2.31.10, BLASTP v2.3.0, InterProScan, tRNAscan-SE v1.3.1, BLASTN v2.3.0 and INFERNAL v1.1.2 were used in genome annotation. BLASTP v2.3.0, MCscan v0.8, MUSCLE, PAML v4.8, OrthoMCL v5, PRANK and PhyML v3.0 were used in whole genome duplication identification. OrthoFinder v2.2.3, Clustal Omega v1.2.4, TrimAl v1.2, RAxML v8.2.11, ASTRAL v5.6.1, PAML MCMCTREE, BLASTP v2.3.0 and Café v4.0.1 were used in phylogenetic analysis. BWA v0.7.17, SAMtools v1.3.1, GATK v3.2.2, SNPEFF, TreeBeST v1.9.2, RAXML v8.2.11, PLINK v1.07, FRAPPE v1.1, ADMIXTURE v1.3.0, EIGENSOFT v3.2 and R were used in population structure analysis.

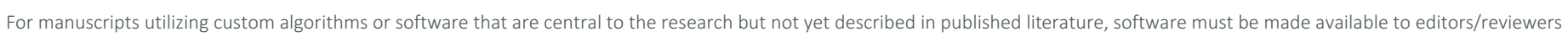

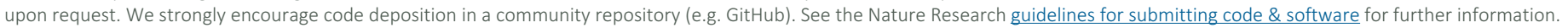


Policy information about availability of data

All manuscripts must include a data availability statement. This statement should provide the following information, where applicable:

- Accession codes, unique identifiers, or web links for publicly available datasets

- A list of figures that have associated raw data

- A description of any restrictions on data availability

Raw reads and genome assembly have been deposited as a BioProject under accession PRJNA418360. Resequencing data have been deposited as a BioProject under accession PRJNA418361.

\section{Field-specific reporting}

Please select the best fit for your research. If you are not sure, read the appropriate sections before making your selection.

$\bigotimes$ Life sciences $\quad \square$ Behavioural \& social sciences

For a reference copy of the document with all sections, see nature.com/authors/policies/ReportingSummary-flat.pdf

\section{Life sciences}

\section{Study design}

All studies must disclose on these points even when the disclosure is negative.

Sample size The size of F1 progenies, i.e., 150 individuals, in Liriodendron is in the standard for linkage map construction.

Data exclusions The reads with low quality are more likely to contain errors, which might complicate the following assembly process, and were excluded. Detailed criteria were provided in Supplementary Note 1.3.

Replication The phenotypic characteristics of Liriodendron chinense were identified independently more than ten times.

Randomization All samples were treated the same and no randomization was performed.

Blinding The Liriodendron genome were sequenced and assembled with no blinding. All seqeuncing data came from the same adult tree; therefore blinding is not relevant to these analyses.

\section{Materials \& experimental systems}

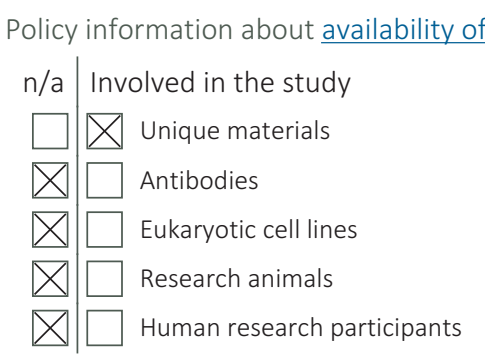

Unique materials

Obtaining unique materials

All Liriodendron individuals used in this study were planted in a forest farm of Nanjing Forestry University, China. Please contact authors for further information.

\section{Method-specific reporting}

$\mathrm{n} / \mathrm{a} \mid$ Involved in the study

Х ChIP-seq

$\square$ Ælow cytometry

$\bigotimes \square$ Magnetic resonance imaging 
Plots

Confirm that:

The axis labels state the marker and fluorochrome used (e.g. CD4-FITC).

The axis scales are clearly visible. Include numbers along axes only for bottom left plot of group (a 'group' is an analysis of identical markers).

$\square$ All plots are contour plots with outliers or pseudocolor plots.

A numerical value for number of cells or percentage (with statistics) is provided.

Methodology

Sample preparation

Yong leaves of this Liriodendron individual used for the whole genome sequencing together with young leaves of Vinca major

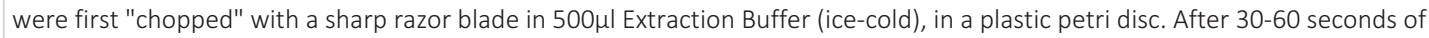
incubation, $2.0 \mathrm{ml}$ Staining Buffer is added. This buffer contains Propidium lodide (PI) as fluorescent dye and RNA-se. To the buffer is also added 0,1\% DTT (Dithiothreitol) and 1\% Polyvinylpyrolidone.

Instrument

Flowcytometer: CyFlow Space (Partec GmbH, Otto Hahnstrasse 32, D-4400 Münster, Germany) with 50 mW, 532 nm green laser

Software

Flomax version 2.8 (Partec)

Cell population abundance

The copped solution, containing cell constituents and large tissue remnants, is passed through a nylon filter of $50 \mu \mathrm{m}$ mesh size. After incubation of at least 30 minutes at room temperature, the filtered solution with stained nuclei is send through the flow cytometer CyFlow (Sysmex Partec GmbH). At least 3000 nuclei of the sample and the internal standard (Vinca major) were measured.

Gating strategy

No specific gating strategy was applied. The peaks of the nuclei were not disturbed by the noise signals.

Tick this box to confirm that a figure exemplifying the gating strategy is provided in the Supplementary Information. 